\title{
Effects of a Nurse-Led Educational Intervention for Chinese Adult Patients with Psoriatic Arthritis: A Case-Control Study
}

\author{
Bishan Cai ${ }^{*}$, Tiantian Xin ${ }^{2}$, Aizhen Yan ${ }^{3}$, Luna Wu ${ }^{1}$, Li Wang1 \\ ${ }^{1}$ Physical Therapy Center, Guangdong Provincial Center for Skin Disease and STIs Control and Prevention \\ (Guangdong Provincial Dermatology Hospital), Guangzhou, China \\ ${ }^{2}$ Department of Dermatology, The Third Affiliated Hospital of Guangzhou Medical University, Guangzhou, China \\ ${ }^{3}$ Inpatient Unit, Guangdong Provincial Center for Skin Disease and STIs Control and Prevention (Guangdong \\ Provincial Dermatology Hospital), Guangzhou, China \\ Email: ${ }^{*}$ caibsnurse@126.com
}

Received 4 January 2016; accepted 19 February 2016; published 22 February 2016

Copyright (C) 2016 by authors and Scientific Research Publishing Inc.

This work is licensed under the Creative Commons Attribution International License (CC BY).

http://creativecommons.org/licenses/by/4.0/

c) (7) Open Access

\begin{abstract}
The aim of this study was to evaluate the effect of an individual nurse-led educational intervention for patients with psoriatic arthritis (PsA). This was a case-control study. The case group consisted of six individual educational sessions delivered by a nurse. A total of 40 patients with PsA joined in this study: the case group $(n=20)$ and the control group $(n=18)$. After a 6 -week intervention, the case group had significantly better management for the severity of arthritis symptoms $(p<0.05)$, better psychological well-being and significant lower levels of anxiety $(p<0.05)$, and depression score ( $p<0.01$ ), and reported better improvement of physical and psychological domain of quality of life (QOL) (both p values < $\mathbf{0 . 0 5}$ ) than the control group. In conclusion, this nurse-led individual education intervention has statistically significant benefits for the management of clinical symptoms of arthritis and for psychological well-being and QOL in patients with PsA.
\end{abstract}

\section{Keywords}

Nurse-Led Interventions, Psoriatic Arthristis, Chinese Patients, Case-Control Study

\section{Introduction}

Psoriatic arthritis (PsA) is a chronic inflammatory arthritis condition [1]. It is a long-term condition that can

\footnotetext{
*Corresponding author.
}

How to cite this paper: Cai, B.S., Xin, T.T., Yan, A.Z., Wu, L.N. and Wang, L. (2016) Effects of a Nurse-Led Educational Intervention for Chinese Adult Patients with Psoriatic Arthritis: A Case-Control Study. Open Journal of Nursing, 6, 110-114. 
have a significant psychological effect on the patient's life [2]. If left untreated, patients with psoriatic arthritis may suffer pain, reduced quality of life (QOL), joint damage, and disability [1]. Patients with psoriatic arthritis are also at higher risk for psychological distress of anxiety and depression [1]. PsA affects men and women equally (typically those between the ages of 30 and 55) [3]. The estimated prevalence of PsA in patients with psoriasis is $30 \%-40 \%$ [4].

Effective patient education for those diagnosed with psoriatic arthritis is essential [5]. In studies on the effect of patient education for patients with PsA, the majority of the interventions have been delivered to groups of patients and less in a one-to-one format [6]. Research evidence shows that patient outcomes may be improved through individual nursing interventions [7]. Previous studies find that patients with arthritis are mostly preferred to individual nurse-delivered education by one-to-one communication on certain themes [8] [9]. While existing studies [10]-[12] investigate the effects of nursing education on the management of psoriasis, these studies do not focus on the specific type of psoriasis, psoriatic arthritis, and may be failed to include the educational component of how to manage symptoms of arthritis. Therefore, the aim of this study is to evaluate the effect of an individual nurse-led educational intervention for patients with PsA.

\section{Methods}

\subsection{Study Design and Sample}

The study design was a case-control study. Inclusion criteria included Chinese adult patients (aged 18 or older) with a diagnosis of psoriatic arthritis, duration of psoriasis with 5 years or longer (patients with psoriasis developed into psoriatic arthritis need at least 5 years). According to the pre-agreed study protocol, in order to achieve a 1:1 ratio in the intervention and control groups, patients were chosen based on age (within 3 years), gender, and duration of psoriasis at diagnosis (within 2 years), in order to form the matched control group. Initially, there were a total of 66 patients with psoriatic arthritis approached: 62 were willing to participate and four declined. Patients who accepted the offer of a nurse-led educational program formed the intervention group $(\mathrm{n}=$ 22). The control group was selected from the 40 volunteer patients who would receive this educational intervention at the end of study periods (6-week post intervention assessment). In order to match demographic characteristics of subjects in the intervention and comparator groups, patients in the control group were chosen based on age, gender, duration and age onset of psoriasis in years $(n=18)$.

\subsection{Nurse-Led Educational Intervention}

The intervention group received an individual nurse-led educational intervention. All participants in both groups received usual care. The intervention was an educational program and the nurses who delivered the program. The intervention consisted of 6 sessions, one week per session. The educational components of this education intervention comprised disease progression of PsA, types of medications and how to observe side effects, how to cope with pain or other symptoms, advices on exercise and nutrition.

\subsection{Outcome Measures}

Intervention effectiveness of clinical severity of psoriasis was assessed by the Psoriasis Area and Severity Index (PASI), which is the most extensively studied psoriasis clinical severity score and the most thoroughly validated [13]. High scores indicated more severity of arthritis. The clinical severity of arthritis was assessed by a numeric rating scare from 0 to 4 in the domains of joint pain, tenderness, swelling and stiffness [14]. High scores indicated more severity of arthritis. The psychological symptoms of anxiety and depression were measured by Self-rating Anxiety Scale (SAS) [15] and Self-rating Depression Scale (SDS) [16]. Lower scores indicate lower levels of anxiety and depression. The QOL of patients with psoriatic arthritis was measured by the World Health Organization Quality of Life-Brief Form (WHOQOL-BREF), which comprises four specific domains: physical, psychological, social relationship and environment. Higher scores indicate better QOL [17].

\subsection{Data Collection and Analysis}

This study was undertaken at Guangdong Provincial Dermatology hospital in South China from January to December 2015. Ethical approval was obtained from the ethics review committee of the studied hospital. All patients participated on a voluntary basis and gave written informed consent before data collection. A staff nurse 
was responsible for recruiting and collecting the outcome. All data were collected by a staff nurse with research training. Data were collected from January to December 2015. SPSS version 20.0 was used for statistical. Comparison analysis of the data was used the Chi-Square test or the independent samples t-test. All statistical tests were two-tailed, and $\mathrm{p}<0.05$ was taken to indicate statistical significance.

\section{Results}

A total of 60 eligible patients were allocated into two groups according to their willingness, 22 in the intervention group and 18 in the control group. Table 1 shows the demographic characteristics of patients in both groups. The mean age in the intervention group was 42.7 years (Standard Deviation, SD = 7.7). The mean age in the control group was 43.1 years $(\mathrm{SD}=7.1)$. More than half of patients were male $(72.7 \%$ in the intervention group, and $72.2 \%$ in the control group). The mean duration of psoriasis was nearly 20 years in both groups. The mean age onset of psoriasis was 22.7 years $(S D=14.2)$ in the intervention group and 23.3 years $(S D=14.2)$ in the control group. All demographic characteristics had no statistically significant differences between the case and control group.

From Table 2, the nurse-led educational intervention has positive effects for clinical severity of arthritis at post-intervention assessment $(p<0.05)$. In addition, the nurse-led education interventions have significant effects for reducing the symptoms of anxiety and depression in the case group ( $p<0.05$, and $p<0.01$, respectively) (Table 3). In terms of the improvement of QOL, this intervention has positive effects for the enhancement of physical and psychological domain of QOL (both p values < 0.05) (Table 4).

\section{Discussion}

Consistent with previous studies [10] [11], the effects of a nurse-led interventions was positive, especially in psychological and QOL outcomes, although the effectiveness of psycho-educational interventions for the physic-

Table 1. Demographic characteristics of participants.

\begin{tabular}{ccc}
\hline Variables & Case $(\mathrm{n}=22)$ & Control $(\mathrm{n}=18)$ \\
\hline Age (years), mean \pm SD & $42.7(7.7)$ & \\
\hline Gender $(\mathrm{n}, \%)$ & $16(72.7)$ & $43.1(7.1)$ \\
\hline Male & $6(27.3)$ & $5(27.8)$ \\
Female & $20.6(11.2)$ & $13.2(11.1)$ \\
Duration of psoriasis (years), mean \pm SD & $22.7(14.2)$ & $23.3(12.4)$ \\
\hline
\end{tabular}

Table 2. Comparison of clinical severity of psoriasis and arthritis symptoms.

\begin{tabular}{|c|c|c|}
\hline Variables & Case $(n=22)$ & Control $(n=18)$ \\
\hline Clinical severity of psoriasis by PASI score at pre-intervention & $21.45(10.52)$ & $20.35(10.26)$ \\
\hline Clinical severity of psoriasis by PASI score at post-intervention & $2.69(1.76)$ & $2.03(1.71)$ \\
\hline Clinical severity of arthritis score at pre-intervention & $8.45(1.56)$ & $8.39(1.64)$ \\
\hline Clinical severity of arthritis score at post-intervention & $1.25(1.06)$ & $3.19(1.15)^{*}$ \\
\hline
\end{tabular}

${ }^{*} \mathrm{P}<0.05$. Abbreviation: PASI, Psoriasis Area and Severity Index.

Table 3. Comparison of anxiety and depression score.

\begin{tabular}{ccc}
\hline Variables & Case $(\mathrm{n}=22)$ & Control $(\mathrm{n}=18)$ \\
\hline Self-rating Anxiety Scale score at pre-intervention & $44.68(7.57)$ & $44.42(8.31)$ \\
Self-rating Anxiety Scale score at post-intervention & $40.05(9.46)$ & $43.95(13.62)^{*}$ \\
Self-rating Depression Scale score at pre-intervention & $41.46(9.22)$ & $41.37(8.67)$ \\
Self-rating Depression Scale score at post-intervention & $37.25(11.02)$ & $41.04(11.47)^{* *}$ \\
\hline
\end{tabular}

\footnotetext{
${ }^{*} \mathrm{P}<0.05,{ }^{* *} \mathrm{P}<0.01$.
} 
Table 4. Comparison of QOL score.

\begin{tabular}{ccc}
\hline Variables & Case $(\mathrm{n}=22)$ & Control $(\mathrm{n}=18)$ \\
\hline Physical QOL score at pre-intervention & $12.65(1.35)$ & $12.86(1.45)$ \\
Physical QOL score at post-intervention & $14.78(1.86)$ & $13.48(1.34)^{*}$ \\
Psychological QOL score at pre-intervention & $11.64(1.52)$ & $12.06(1.12)$ \\
Psychological QOL score at post-intervention & $13.89(1.96)$ & $12.81(1.45)^{*}$ \\
Social QOL score at pre-intervention & $12.57(1.66)$ & $12.47(1.74)$ \\
Social QOL score at post-intervention & $12.87(1.44)$ & $12.78(1.31)$ \\
Environmental QOL score at pre-intervention & $10.45(1.46)$ & $10.68(1.34)$ \\
Environmental QOL score at post-intervention & $10.88(1.52)$ & $10.52(1.42)$ \\
\hline
\end{tabular}

${ }^{*} \mathrm{P}<0.05$. Abbreviation: QOL, quality of life.

cal severity of psoriasis was inconsistent. This study uniquely found that the nurse-led educational intervention has positive effects on reducing the clinical severity scores of arthritis. This individual education intervention shows positive effects in improving the symptom management of arthritis, psychological well-being and QOL. The reason for designed this individual intervention was offering the opportunity to focus on individual learning needs in one-to-one communication with a nurse [6]. And effective patient education is essential to ensure individuals have a good understanding of their own conditions, which in turn will improve adherence with their individual management plan [5].

Nurse delivered interventions have the opportunity to focus on individual learning needs [8]. By an individual delivery format, this nurse-led education intervention may be able to confirm that the patients are being individualized to each patient's health experience [14]. Findings of this study were consistent with a review of effects of psycho-education interventions for the management of psoriasis [18].

Dermatology nurses play an important role in screening and assessment of patients with early PsA. Active monitoring of psoriasis patients for signs of joint or arthritic involvement and familiarity with PsA screening, diagnosis and treatment options can help dermatologists positively impact the clinical course of psoriatic disease [19]. As PsA is a long-term and impact both cutaneous and joint disease, a multidisciplinary approach to support patients for the management of PsA is needed. Effective patient education as well as regular assessment of the PsA by nurses could have additional benefits to ensure the efficacy of treatments [18].

There is a couple of study limitation. Firstly, due to practical limitation, it is difficult to blind patients to the fact that they are taking part in an educational intervention. Secondly, this study was only assessed the intervention effect at immediate post-interventions. Future research should investigate whether the benefits are sustained in a longer follow-up period.

\section{Conclusion}

This nurse-led individual education intervention has statistically significant benefits for the management of clinical symptoms of arthritis and for psychological well-being and QOL in patients with PsA.

\section{References}

[1] Dewing, K.A. (2015) Management of Patients with Psoriatic Arthritis. The Nurse Practitioner, 40, 40-46. http://dx.doi.org/10.1097/01.NPR.0000461950.23292.18

[2] Chang, C.A., Gottlieb, A.B. and Lizzul, P.F. (2011) Management of Psoriatic Arthritis from the View of the Dermatologist. Nature Reviews Rheumatology, 7, 558-598. http://dx.doi.org/10.1038/nrrheum.2011.125

[3] Bruce, I.N. (2008) Psoriatic Arthritis: Clinical Features. In: Hochberg, M.C., Silman, A.J., Smolen, J.S., Weinblatt, M.E. and Weisman, M.H., Eds., Rheumatology, 4th Edition, Elsevier, Philadelphia, 989-997.

[4] Cobo-Ibáñez, T., Villaverde, V., Seoane-Mato, D., et al. (2015) Multidisciplinary Dermatology-Rheumatology Management for Patients with Moderate-to-Severe Psoriasis and Psoriatic Arthritis: A Systematic Review. Rheumatology International, in press.

[5] Waldron, N. (2012) Care and Support of Patients with Psoriatic Arthritis. Nursing Standard, 26, 35-39. http://dx.doi.org/10.7748/ns2012.08.26.52.35.c9247 
[6] Grønning, K., Skomsvoll, J.F., Rannestad, T. and Steinsbekk, A. (2012) The Effect of an Educational Programme Consisting of Group and Individual Arthritis Education for Patients with Polyarthritis-A Randomised Controlled Trial. Patient Education and Counseling, 88, 113-120. http://dx.doi.org/10.1016/j.pec.2011.12.011

[7] Suhonen, R., Valimaki, M. and Leino-Kilpi, H. (2008) A Review of Outcomes of Individualized Nursing Interventions on Adult Patients. Journal of Clinical Nursing, 17, 843-860. http://dx.doi.org/10.1111/j.1365-2702.2007.01979.x

[8] Makelainen, P., Vehvilainen-Julkunen, K. and Pietila, A.M. (2009) Rheumatoid Arthritis Patient Education: RA Patients' Experience. Journal of Clinical Nursing, 18, 2058-2065. http://dx.doi.org/10.1111/j.1365-2702.2008.02763.x

[9] Barlow, J.H., Cullen, L.A. and Rowe, I.F. (2002) Educational Preferences, Psychological Wellbeing and Self-Efficacy among People with Rheumatoid Arthritis. Patient Education and Counseling, 46, 11-19. http://dx.doi.org/10.1016/S0738-3991(01)00146-X

[10] Li, A. (2010) Observation on Effect of Comprehensive Nursing Intervention for Patients with Psoriasis. Chinese Nursing Research, 24, 2125.

[11] Wu. L., Sun, X., Qiu, M. and Feng, Z. (2010) Effects of Psychological Interventions on Psychological Health of Patients with Psoriasis. Chinese Modern Clinical Nursing, 9, 38-39.

[12] Zhang, J., Deng, Y., Ye, T., et al. (2011) Investigation of Effects of Chinese Herbal Medicine Combined with Psychological Interventions on 42 Cases of Patients with Psoriasis. Journal of New Chinese Medicine, 43, 61-62.

[13] Puzenat, E., Bronsard, V., Prey, S., et al. (2010) What Are the Best Outcome Measures for Assessing Plaque Psoriasis Severity? A Systematic Review of the Literature. Journal of the European Academy of Dermatology and Venereology, 24, 10-16. http://dx.doi.org/10.1111/j.1468-3083.2009.03562.x

[14] Moll, J.W. and Wright, V. (1973) Psoriatic Arthritis. Seminars in Arthritis and Rheumatism, 3, 55-78. http://dx.doi.org/10.1016/0049-0172(73)90035-8

[15] Zung, W.W.K. (1971) A Rating Instrument for Anxiety Disorders. Psychosomatics, 12, 371-379. http://dx.doi.org/10.1016/S0033-3182(71)71479-0

[16] Zung, W.W.K. (1965) A Self-Rating Depression Scale. Archives of General Psychiatry, 12, 63-70. http://dx.doi.org/10.1001/archpsyc.1965.01720310065008

[17] World Health Organization (2009) Addressing Global Mental Health Challenges. Switzerland, Geneva.

[18] Chen, R.F., Xin, T.T. and Cheng, A.S.K. (2014) Evaluating the Effectiveness of Psychological and/or Educational Intervenetions in Psoriasis: A Narrative Review. Journal of Dermatology, 41, 775-778. http://dx.doi.org/10.1111/1346-8138.12583

[19] Mease, P.J. and Armstrong, A.W. (2014) Managing Patients with Psoriatic Disease: The Diagnosis and Pharmacologic Treatment of Psoriatic Arthritis in Patients with Psoriasis. Drugs, 74, 423-441. http://dx.doi.org/10.1007/s40265-014-0191-y 